\title{
LES ANOPHÈleS ET LA TRANSMISSION DU PALUDISME À AMBOHIMENA, VILLAGE DE LA MARGE OCCIDENTALE DES HAUTES-TERRES MALGACHES
}

\author{
RAJAONARIVELO V.*, LE GOFF G.*, COT M.** \& BRUTUS L.**,***
}

Summary: ANOPHELINES AND MALARIA TRANSMISSION IN AMBOHIMENA, A VILlage OF the OCCIDENTAL fRINGE OF Madagascar Highlands

The anopheline vectors and malaria transmission were investigated in the Middle West of Madagascar, in the village of

Ambohimena (at the altitude of 940 meters) during two years (August 1996 to July 1998). This village is located outside the vector control area, where yearly DDT house spraying campaigns have been conducted between 1993 and 1998. Collection of mosquitoes was mainly based on all night man biting collections (650 man-nights), pyrethrum spray catches (224 bedrooms) and direct collections in outdoor resting places (140 toilets,

61 pigsties, 33 holes, 19 sheds, 79 sisal hedges, 70 cart shelters). Blood fed anophelines allowed analysis of the origin of blood with an ELISA method. Presence of circum-sporozoite protein was assessed with another ELISA method. The total number of collected anophelines was 14,280. Two malaria vectors were identified: Anopheles funestus Giles, 1900 and An. arabiensis Patton, 1902. An. funestus was the most abundant mosquito, especially during the hot rainy season. Two peaks of abundance were observed (in December and April). Endophagic rate (for mosquitoes aggressive for man) of $35.3 \%$, an endophilic rate (for resting mosquitoes) of $78.0 \%$ and an anthropophilic rate (for indoor resting mosquitoes) of $64.0 \%$ were calculated. The average parity rate was relatively low (61.2\%). The Plasmodium falciparum immunological sporozoite rate was $0.20 \%$.

An. funestus presented a higher vectorial capacity during the first round of rice cultivation (January) than during the second round (April-May). An. arabiensis was mostly abundant in December and January at the beginning of the rainy season. This species was exophagic (endophagic rate $=27.5 \%$ ) and zoophilic (anthropophilic rate $=7.8 \%$ ). The sporozoitic index was determinated as zero (number of examined mosquitoes $=871$ ). In this village, An. arabiensis presented only marginal importance for malaria transmission. Malaria transmission occurred from December to April. Annual entomological inoculation rate, only due to An. funestus, was 8.96 during the first year, and 3.17 during the second year. In this area where transmission is moderately stable, we suggest an extension of vector imagocidal control activities up to the western fringes of the Highlands.

KEY WORDS : malaria, Anopheles funestus, Anopheles arabiensis, transmission, Madagascar

\footnotetext{
* Institut de Recherche pour le Développement, UR 16 Caractérisation et contrôle des populations de vecteurs, LIN-IRD, BP 64501, 911, av. Agropolis, 34394 Montpellier Cedex 5, France. E-mail: legoff@mpl.ird.fr

** IRD, UR 10 Santé de la mère et de l'enfant, Paris, France.

*** INLASA, La Paz, Bolivie.

Correspondance : Voahirana Rajaonarivelo, Laboratoire des Insectes Nuisibles, IRD, BP 64501, 911, avenue Agropolis, 34394 Montpellier Cedex 5, France.

Tél. : + $33(0) 467043223$ - Fax : + $33(0) 467542044$

E-mail : nirajao@yahoo.fr
}

\begin{abstract}
Résumé :
Une étude a été menée dans le Moyen-Ouest de Madagascar à 940 mètres d'altitude dans le village d'Ambohimena. Ce village est situé en dehors de la zone d'aspersions intra-domiciliaires d'insecticides rémanents. Deux vecteurs, An. funestus Giles, 1900 et An. arabiensis Patton, 1905 y sont présents. An. funestus est abondant au cours de la saison chaude et humide, avec deux pics d'abondance en décembre et avril. L'endophagie d'An. funestus est faible (taux d'endophagie $=35,3 \%$ ). C'est une espèce endophile (taux d'endophilie $=78 \%$ ) et anthropophile (taux d'anthropophilie $=64 \%$ ). Son infectivité est faible (taux d'infectivité $=0,20 \%$. Le taux d'inoculation spécifique de Plasmodium falciparum par l'espèce An. funestus a été de moins de 10 piqûres par homme et par an. Cette espèce présente une capacité vectrice maximale à la fin du premier tour de riz (janvier) alors que son abondance maximale est observée à la fin du deuxième tour (avrit-mai). An. arabiensis est abondant entre décembre et janvier et est lié à la présence des gîtes pluviaux favorables au développement larvaire. C'est une espèce exophage (taux d'endophagie $=27,5 \%$ ) et zoophile (taux $d^{\prime}$ anthropophilie $=7,8 \%$ ). An. arabiensis joverait un rôle secondaire dans la transmission du paludisme car la présence des plasmodies n'a pas été mise en évidence (effectif testé $=871$ ). Dans ce village, la stabilité du paludisme est modérée ou intermédiaire; plus proche de l'instabilité que de la stabilité avérée. Il est suggéré que la lutte antivectorielle soit étendue aux zones de transition stabilité-instabilité qui constitueraient un réservoir de vecteurs et de plasmodies, afin de prévenir l'émergence de nouvelles épidémies sur les Hautes-Terres.
\end{abstract}

MOTS CLÉS : paludisme, Anopheles funestus, Anopheles arabiensis, transmission, Madagascar.

\section{INTRODUCTION}

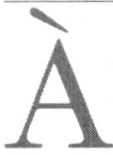

Madagascar, le climat et le relief, et plus précisément les précipitations et la température, influencent considérablement le rythme et l'intensité de la transmission du paludisme. Les variations climatiques déterminent quatre grands faciès épidémiologiques (Mouchet et al., 1995) :

- le faciès équatorial avec une transmission permanente ou subpermanente, principalement sur la côte est;

- le faciès tropical avec une transmission saisonnière interrompue par la saison sèche, principalement dans l'ouest de l'île;

- le faciès des Hautes-Terres avec une transmission sai- 
sonnière interrompue par la saison sèche et froide, principalement dans la zone centrale du pays;

- et le faciès du sud, sub-aride, avec une transmission saisonnière courte, principalement dans l'extrême sud et dans le sud-ouest.

Les Hautes-Terres centrales couvrent environ $20 \%$ de la superficie de l'île et concernent une zone densément peuplée des provinces d'Antananarivo et de Fianarantsoa. Ces Hautes-Terres ont servi de cadre à une Opération de pulvérisation intra-domiciliaire de DDT (OPID) conduite par le Ministère de la Santé de 1993 à 1998 dans les villages situés entre 1000 et 1500 mètres d'altitude. En 1999 et 2000, ces opérations se sont poursuivies, de manière plus localisée, sous le nom de CAID (Campagne d'aspersions intra-domiciliaire).

La présente étude s'est déroulée dans un village traditionnel, typique de la marge occidentale des HautesTerres de Madagascar. Ce travail avait pour buts : d'évaluer la dynamique des populations d'anophèles vecteurs, leur biologie et leur éthologie, et de caractériser la dynamique de la transmission du paludisme. Sur cette base, nous suggérons des solutions visant à améliorer l'impact des actions de lutte anti-vectorielle appliquées à la lutte anti-paludique sur les marges des Hautes-Terres centrales.

\section{LA ZONE D'ÉTUDE}

L'étude s'est déroulée à Ambohimena (altitude 940 mètres, $46^{\circ} 18^{\prime} \mathrm{E}, 19^{\circ} 35^{\prime} \mathrm{S}$ ). Le village se trouve à une centaine de kilomètres à l'ouest d'Antsirabe, dans la commune de Mandoto. Il se situe en haut d'une colline entourée de bas-fonds aménagés pour la riziculture. L'activité principale est l'agriculture. Les cultivateurs pratiquent deux tours de riz : $i$ ) la culture irriguée "vary aloha" qui couvre $25 \%$ de la surface totale des rizières et qui procure une récolte fin décembre-début janvier et ii) la culture pluviale "vary vakiambiaty" qui couvre la totalité des bas-fonds et qui procure une récolte en avril-juin.

Les Hautes-Terres de Madagascar sont situées à des altitudes supérieures à 800 mètres. Le climat y est de type tropical d'altitude. Il existe deux saisons très différentes : une sèche et fraîche de mai à octobre (cinq à six mois) et une saison humide et chaude, de novembre à avril. Les précipitations annuelles sont en moyenne de $1500 \mathrm{~mm}$; pendant les cinq mois les plus secs, il tombe moins de $20 \mathrm{~mm}$ de pluie (mai-septembre). La température moyenne annuelle est de $22^{\circ} \mathrm{C}$ dans la région avoisinant 1000 mètres d'altitude et la moyenne des mois les plus frais, juillet ou août, se situe entre $15^{\circ} \mathrm{C}$ et $18^{\circ} \mathrm{C}$ (Donque, 1975).

Le village d'Ambohimena est situé dans la pénéplaine de Mandoto. Les vents humides d'est soufflent parfois avec violence, particulièrement en fin de saison des pluies. Au contraire, les vents d'ouest, plus secs, soufflent régulièrement en saison sèche.

À Ambohimena, la pression insecticide n'existe plus depuis l'arrêt des pulvérisations vers les années 70. En effet, ce village n'a pas été concerné par l'OPID et est situé à une quinzaine de kilomètres à vol d'oiseau endehors de la zone pulvérisée.

\section{MATÉRIELS ET MÉTHODES}

L e suivi entomologique a été réalisé avec une fréquence mensuelle d'août 1996 à juillet 1998 selon trois méthodes :

- des captures nocturnes des femelles agressives sur homme de 18 h 00 à 06 h 00 du matin. Les moustiques ont été collectés au fur et à mesure qu'ils venaient se poser sur le captureur;

- des captures de moustiques endophiles, le matin, à l'intérieur des habitations. Les moustiques ont été collectés sur des draps disposés par terre à cet effet, après pulvérisation d'un pyréthrinoïde;

- des captures diurnes de moustiques exophiles, à l'extérieur des habitations dans différents lieux de repos (abris à charrettes, porcheries, abris sousvéranda, haies de sisal) et deux puits de MuirheadThomson (1958) situés à l'extérieur du village. Les moustiques ont été recherchés à l'observation directe et collectés à l'aide d'un aspirateur à bouche.

Ces méthodes de capture permettent : d'identifier les espèces culicidiennes agressives pour l'homme; d'évaluer l'agressivité, c'est-à-dire le nombre de piqûres par homme et par nuit (PHN) pour chaque espèce agressive; d'estimer le taux d'endophagie d'une espèce agressive selon que le captureur est placé à l'intérieur ou à l'extérieur des maisons, c'est-à-dire les densités agressives moyennes obtenues à l'intérieur divisées par la somme des densités agressives moyennes à l'intérieur et à l'extérieur, multiplié par 100; le taux d'endophilie qui est le rapport entre le nombre moyen de femelles anthropophiles capturées le matin au repos et la densité moyenne, évaluée la nuit précédente, dans les mêmes maisons, multiplié par 100.

L'identification des moustiques a été réalisée par l'utilisation des clés de détermination de Gillies \& Coetzee (1987) et de Grjebine (1966). Les anophèles du complexe Anopheles gambiae ont été déterminés par PCR (Scott et al., 1993).

Après identification et dénombrement, seuls les vecteurs potentiels ont été disséqués pour les ovaires. La détermination de la parturité a été faite par observation microscopique (grossissement 10 ou 40) des trachéoles ovariennes à l'état sec, selon la méthode de Detinova (1963); ces observations n'ont été effectuées qu'au cours de la deuxième année d'étude (juillet 
1997-août 1998). L'observation de l'état de réplétion des femelles capturées sur homme et des femelles au repos nous a permis de définir la proportion des femelles qui présentaient un stade de développement ovarien avancé (semi-gravide, sub-gravide et gravide) et qui avaient besoin de prendre un deuxième repas de sang pour accomplir leur cycle trophogonique complet.

La tête et le thorax des vecteurs potentiels ont été conservés individuellement dans un tube étanche contenant du silicagel. Ils ont été testés par ELISA-CSP (Enzyme-Linked Immunosorbent Assay-Circum Sporozoite Protein) pour la détection et la spéciation des antigènes cirçum-sporozoïtaires (Burkot et al., 1984; Wirtz et al., 1987). Cette méthode a permis d'évaluer l'indice sporozoïtique immunologique spécifique de l'espèce Plasmodium falciparum.

Les abdomens des moustiques gorgés, récoltés au repos, ont été écrasés sur papier-filtre. Les spots de sang ont été gardés au frais et à sec dans une boîte contenant du silicagel. Ces spots ont été testés en ELISA pour identification des origines du repas sanguin (homme, bœuf) selon la méthode de Beier et al., 1988; Burkot et al., 1981. Seuls les résultats des analyses de la deuxième année d'étude (juillet 1997-août 1998) sont présentés dans cet article.

Différents paramètres épidémiologiques ont été utilisés pour caractériser la transmission du paludisme : le taux de parturité (rapport du nombre de femelles pares sur le nombre de femelles observées) ; le taux quotidien de survie $\mathrm{p}$ (racine $\mathrm{L}^{\text {ième }}$ de la parturité; L étant la durée du cycle trophogonique, comprise entre 2,5 et trois jours selon le vecteur considéré et les saisons); l'indice d'anthropophilie (proportion de repas de sang pris sur homme); la capacité vectorielle comme proposée par Garett-Jones (1964) et l'indice de stabilité qui dépend de l'anthropophilie et de la longévité des vecteurs (MacDonald, 1957). La transmission a été calculée comme le taux d'inoculation entomologique, en multipliant la densité agressive moyenne par l'infectivité du vecteur considéré.

Les analyses statistiques ont été faites à l'aide du test $\chi^{2}$ de Mantel-Haenszel ou des probabilités exactes de Fisher (logiciel EPIInfo, version 5.01)

\section{RÉSULTATS}

u cours des 64 nuits de capture, un total de
650 hommes-nuits de capture ont été effectués
(dont 356 à l'intérieur et 294 à l'extérieur). La capture de moustiques au repos par pyréthrage a concerné 224 chambres et 98 débarras. La capture dans les puits de Muirhead-Thomson a concerné 48 puitsnuits au total. Les gîtes extérieurs ont été inventoriés par catégories ( 61 porcheries, 79 haies de sisal, 140 toilettes, 70 abris de charrettes, 64 abris sous-véranda, 19 maisons abandonnées et 33 trous artificiels ou naturels). Au total 15439 moustiques femelles ont été capturés dont $46,1 \%$ agressifs pour l'homme et $53,9 \%$ au repos.

Au sein de la sous-famille des Culicinae, les espèces collectées appartenaient à quatre genres : Culex, Aedes, Mansonia et Coquilletidia (tableau I). Les anophèles ont de loin été les moustiques les plus abondants (92,5\% des Culicidae). An. funestus et An. gambiae s. 1. sont les seuls vecteurs potentiels présents sur les Hautes-Terres malgaches; An. mascarensis De Meillon,

\begin{tabular}{|c|c|c|c|c|}
\hline \multirow[b]{2}{*}{ Espèces ou genres } & \multicolumn{2}{|c|}{ Moustiques agressifs pour l'homme } & \multicolumn{2}{|c|}{ Moustiques capturés au repos } \\
\hline & Intérieur & Extérieur & Maisons d'habitation* & Autres** \\
\hline An. funestus & $1741(82,7)$ & $2634(52,6)$ & $4057(95,4)$ & $2429(59,7)$ \\
\hline An. arabiensis & $72(3,4)$ & $157(3,1)$ & $126(3,0)$ & $576(14,2)$ \\
\hline An. coustani & $53(2,5)$ & $793(15,8)$ & $1(<0,1)$ & $36(0,9)$ \\
\hline An. mascarensis & $37(1,7)$ & $359(7,2)$ & $12(0,3)$ & $436(10,7)$ \\
\hline An. squamosus & $25(1,2)$ & $158(3,2)$ & $1(<0,1)$ & $2(<0,1)$ \\
\hline An. rufipes & $20(0,9)$ & $66(1,3)$ & $9(0,2)$ & $327(8,0)$ \\
\hline An. maculipalpis & $10(0,5)$ & $38(0,8)$ & $0(0,0)$ & $83(2,1)$ \\
\hline An. pretoriensis & $4(0,2)$ & $18(0,4)$ & $0(0,0)$ & $0(0,0)$ \\
\hline Anopheles & $1962(93,2)$ & $4223(84,2)$ & $4206(98,9)$ & $3889(95,6)$ \\
\hline Culex & $86(4,1)$ & $546(10,9)$ & $37(0,9)$ & $160(3,9)$ \\
\hline Mansonia & $46(2,2)$ & $210(4,2)$ & $9(0,2)$ & $12(0,3)$ \\
\hline Aedes & $6(0,3)$ & $27(0,5)$ & $0(0,0)$ & $6(0,1)$ \\
\hline Coquilletidia & $4(0,2)$ & $10(0,2)$ & $0(0,0)$ & $0(0,0)$ \\
\hline Effectifs capturés & 2104 & 5016 & 4252 & 4067 \\
\hline
\end{tabular}

* Maisons d'habitations : chambre à coucher et débarras.

** Abris à charrettes, porcherie, abris et trous divers, puits de Muirhead-Thomson, toilettes, sisal, etc.

Tableau I. - Effectifs et proportions (en \%) des espèces anophéliennes et des genres culicidiens par méthode de capture et par lieu de repos. 
1947 n'ayant à ce jour été trouvé vecteur que dans des zones côtières (Fontenille \& Campbell, 1992; Marrama et al., 1999). De ce fait, notre étude sur la biologie des anophèles n'a pris en compte que ces deux groupes d'espèces.

\section{ANOPHELES FUNESTUS}

L'agressivité moyenne de cette espèce a été de 6,73 piqûres par homme et par nuit $(n=4375)$, soit 2458 piqûres par homme et par an. Elle a été 2,5 fois plus élevée en saison humide (9,73 PHN) qu'en saison sèche (3,71 PHN). Les densités agressives moyennes ont été de 4,89 PHN à l'intérieur et de 8,96 PHN à l'extérieur. Le taux d'endophagie moyen a été de $35,3 \%$. En moyenne, 13,4\% des femelles agressives pour l'homme présentaient un stade ovarien avancé (semi-gravide, sub-gravide ou gravide). Ces pourcentages étaient respectivement de 16,1\%(n=1126) et $9,7 \%(n=825)$ pour les moustiques qui piquaient à l'intérieur et à l'extérieur des habitations; cette différence est significative (test $\chi^{2}, p=4,35.10^{-5}$ ). Que ce soit pour les captures endophages ou exophages, les proportions de femelles qui prenaient un deuxième repas de sang ne présentent pas de différence entre la saison sèche et la saison humide.

Les femelles d'An. funestus ont été agressives durant toute la nuit, particulièrement au milieu de la nuit pour les moustiques endophages (de 23 heures à 5 heures du matin) et, pour les moustiques exophages, un pic d'agressivité entre 2 et 4 heures du matin. Cette espèce a été présente toute l'année avec deux pics d'abondance observés au début et à la fin de la saison chaude tant pour les moustiques endophages qu'exophages (fig. 1).

Au total, 6486 An. funestus ont été capturés au repos, dont 31,8\% dans les chambres à coucher, 30,7\% dans les débarras et $37,5 \%$ à l'extérieur des habitations humaines.

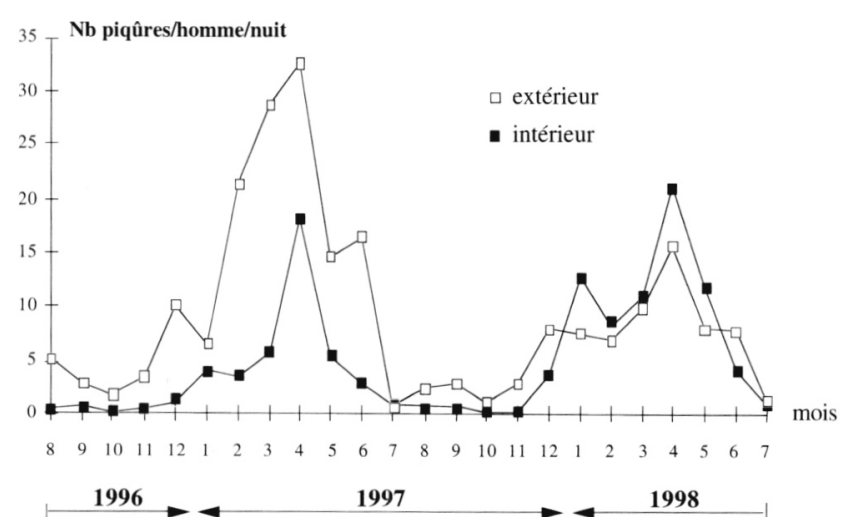

Fig. 1. - Évolution mensuelle des densités agressives d'An. funestus à l'intérieur et à l'extérieur des habitations à Ambohimena (Mandoto) d'août 1996 à juillet 1998.
Le taux d'endophilie a été en moyenne de $78 \%$ mais avec de fortes variations mensuelles. Ce taux d'endophilie a été plus élevé au début de la saison chaude et humide qu'à la fin de cette saison.

La proportion moyenne de femelles gorgées sur homme a été évaluée à 40,0\% $(n=2436)$ mais avec de considérables variations d'un site de capture à un autre. Les deux hôtes principaux de la population d'An. funestus dans ce village ont été le zébu (57\%) et l'homme (40\%). Les moustiques capturés dans les chambres ont logiquement présenté une proportion de spécimens gorgés sur homme nettement plus élevée $(64,1 \%)$ que dans les autres gîtes de repos (24,6 \% en débarras et 15,4\% dans les abris extérieurs). Cette proportion a été également variable en fonction des saisons. Elle a été plus élevée en saison chaude (33-37\%) et humide qu'en saison froide et sèche $(19 \%)$; la différence est significative $(\mathrm{p}=$ 0,001). Ainsi, on remarque que plus on s'éloigne de l'environnement humain, plus la proportion de repas pris simultanément sur homme et zébu augmente (9,4\% en chambre à coucher et $29,6 \%$ en gîtes extérieurs).

Le taux de parturité moyen pour An. funestus a été de $61,2 \%(n=1970)$. Il a été de $60,1 \%$ pour les moustiques endophages et de $62,7 \%$ pour les exophages; la différence n'est pas significative $(p=0,228)$. En revanche, une différence significative a été observée entre les taux de parturité en saison sèche $(55,1 \%)$ et en saison humide $(63,4 \%)(p=0,00075)$.

Le taux quotidien de survie pour cette espèce était, respectivement, de $82,2 \%$ et de $84,9 \%$ pour un cycle gonotrophique de 2,5 jours et de trois jours.

L'indice sporozoïtique immunologique spécifique de $P$. falciparum a été globalement de 0,20\% pour l'ensemble des captures (22 positifs sur 10753 testés). Entre les An. funestus capturés sur hommes et ceux récoltés au repos dans les habitations humaines, l'indice sporozoïtique immunologique n'a pas été différent (ISI égal respectivement à 0,25\%, 0,31\% et 0,20\% pour les femelles agressives pour l'homme, les moustiques collectés au repos dans les chambres à coucher et ceux au repos dans les débarras). Ils ont été trouvés infectés uniquement parmi la population en relation directe avec l'homme ou son habitat. En revanche, il est à noter qu'aucune femelle capturée au repos dans les abris extérieurs n'a révélé la présence d'antigène $\operatorname{CSP}(n=2151)$.

En dépit de densités moyennes agressives deux fois plus faibles à la fin du premier tour de riz (ma moyen = 8,28 en décembre 1997 et janvier 1998) comparativement à celles observées à la fin du deuxième tour de riz ( ma moyen $=16,53$ en avril-mai 1998), la capacité vectorielle d'An. funestus à été trois fois plus forte en décembre-janvier (tableau II). Les capacités vectorielles de la fraction endophage et endophile 


\begin{tabular}{|c|c|c|c|c|c|c|}
\hline Espèces & $\begin{array}{l}\text { Système } \\
\text { rizicole }\end{array}$ & Mois & $\begin{array}{l}\text { Densité } \\
\text { moyenne } \\
\text { (ma) }\end{array}$ & $\begin{array}{c}\text { Anthropophilie } \\
(\%) \\
\text { (a) }\end{array}$ & $\begin{array}{c}\text { Taux quotidien } \\
\text { de survie } \\
\text { (p) }\end{array}$ & $\begin{array}{c}\text { Capacité } \\
\text { vectorielle } \\
C^{*}\end{array}$ \\
\hline \multirow[t]{2}{*}{ An. funestus } & $\begin{array}{l}\text { riz irrigué } \\
\text { (vary aloba) }\end{array}$ & $\begin{array}{l}\text { décembre-janvier } \\
\text { épiaison-moisson }\end{array}$ & 8,281 & $\begin{array}{c}84,4 \\
(n=141)\end{array}$ & $\begin{array}{c}0,8679 \\
(\mathrm{n}=238)\end{array}$ & 2,36 \\
\hline & $\begin{array}{c}\text { riz pluvial } \\
\text { (vary vakiambiaty) }\end{array}$ & $\begin{array}{c}\text { avril-mai } \\
\text { épiaison-moisson }\end{array}$ & 16,531 & $\begin{array}{c}50,8 \\
(\mathrm{n}=612)\end{array}$ & $\begin{array}{c}0,8150 \\
(\mathrm{n}=507)\end{array}$ & 0,77 \\
\hline An. arabiensis & - & $\begin{array}{c}\text { janvier } 1998 \\
\text { (densité maximum) }\end{array}$ & 1,88 & $\begin{array}{c}100 \\
(n=2)\end{array}$ & $\begin{array}{c}0,8026 \\
(\mathrm{n}=26)\end{array}$ & 0,16 \\
\hline
\end{tabular}

* Les capacités vectorielles ont été calculées comme suit pour un homme adulte (b), un cycle plasmodial extrinsèque de 15 jours (n) et un cycle gonotrophique de 2,5 jours $(\mathrm{L}): \mathrm{C}=\mathrm{ma}^{2} \mathrm{bp}^{\mathrm{n}} /-\log _{\mathrm{e}} \mathrm{p}$.

Tableau II. - Paramètres entomologiques de la fraction endophile et endophage d'An. funestus et d'An. arabiensis en fonction des récoltes rizicoles et de l'abondance des vecteurs (Ambohimena, 1997, 1998).

d'An. funestus (calculées pour un adulte, un cycle gonotrophique de 2,5 jours et pour un cycle sporogonique de 15 jours) ont été de 2,36 pour la période correspondant à l'épiaison-moisson du "vary aloha" (premier tour de riz, irrigué) et seulement de 0,77 pour le "vary vakiambiaty" (deuxième tour de riz, irrigué et/ou pluvial).

\section{ANOPHELES ARABIENSIS}

L'identification de moustiques du complexe An. gambiae par la méthode PCR a démontré qu'ils appartenaient tous à l'espèce An. arabiensis (D. Fontenille, comm. pers. : 95,3\% d'An. arabiensis sur 337 testés; les $4,7 \%$ restants étant interprétés comme un défaut de la technique de diagnostic ou de conservation d'ADN).

L'agressivité de cette espèce a été de 0,35 piqûres/ homme/nuit $(\mathrm{PHN})(\mathrm{n}=229)$, soit 129 piqûres par homme et par an. Les densités agressives moyennes ont été de 0,20 PHN à l'intérieur et de 0,53 PHN à l'extérieur; ainsi, cette espèce a présenté une nette tendance à l'exophagie. L'endophagie a été de $27,5 \%$. L'agressivité de cette espèce a été la plus importante au cours de la première partie de la nuit. Ce phénomène a été plus net pour les moustiques exophages : le pic d'agressivité a été observé entre 21 et 22 heures. Dans les différents lieux de capture, cette espèce a été essentiellement exophile puisque $82,0 \%$ des spécimens capturés au repos $(\mathrm{n}=702)$ ont été récoltés à l'extérieur des habitations et seulement $4,0 \%$ dans les chambres à coucher et $14,0 \%$ dans les débarras.

De l'analyse des repas de sang, on a déduit que le taux d'anthropophilie global était de 7,8\%. Il a été de $21,6 \%$ pour la fraction endophile (chambres et débarras) et seulement 4,1\% pour la fraction exophile récoltée dans les gîtes extérieurs; cette différence est significative (probabilité exacte de Fisher $\mathrm{p}<10-3$ ). Le taux moyen de parturité était de 25,5\% $(\mathrm{n}=141)$. Il était de $38,3 \%$ pour les moustiques endophages ( $\mathrm{n}=$ 47) et de $19,1 \%$ pour les exophages $(n=94)$; cette différence est significative $(p=0,014)$. Du taux moyen de parturité, on en déduit que le taux quotidien de survie était de $57,9 \%$ pour un cycle gonotrophique de 2,5 jours et de $63,4 \%$ pour un cycle de trois jours.

La capacité vectorielle d'An. arabiensis a été extrêmement faible dans ce village. Elle a été évaluée à 5,5.10 (tableau II). En janvier 1998, soit au maximum de son abondance à l'intérieur des maisons $(\mathrm{ma}=1,88)$ et de son anthropophilie dans les chambres (100\%), sa capacité vectorielle a été seulement de 0,16.

Aucun spécimen d'An. arabiensis testé en ELISA ( $\mathrm{n}=871)$ n'a permis de détecter la présence d'antigène CSP spécifique de l'espèce $P$. falciparum.

\section{TRANSMISSION ET STABILITÉ DU PALUDISME}

La transmission existe durant toute la saison chaude et humide puis elle s'annule durant la saison sèche et froide. C'est une transmission de type saisonnière longue qui a été observée pendant six mois, de décembre à mai (fig. 2). Le premier pic de transmission correspond à la récolte du premier tour de riz (décembre-janvier) et le deuxième pic à la récolte du deuxième tour de riz (avril-mai). Une très forte densité agressive observée en avril et en mai ne correspond pas à des taux d'inoculation très élevés du fait d'une faible infectivité. Le taux d'inoculation annuel

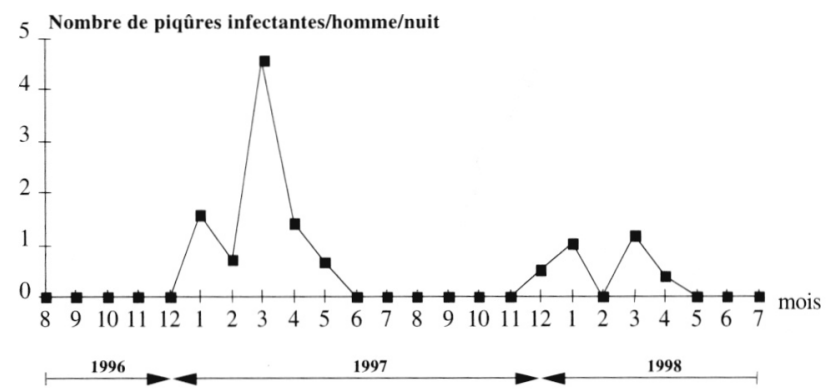

Fig. 2. - Variation mensuelle du taux d'inoculation spécifique de Plasmodium falciparum par Anopheles funestus à Ambohimena (Mandoto) d'août 1996 à juillet 1998. 
observé pour l'espèce $P$. falciparum a été de 8,96 piqûres/homme/an la première année et 3,17 piqûres/ homme/an la deuxième année.

L'indice de stabilité a été de 1,3 au cours de la deuxième année d'étude. Il en résulte que cette zone doit être considérée comme une zone où la stabilité du paludisme est modérée, ou intermédiaire, en étant plus proche de l'instabilité (valeur seuil admise $=0,5$ ) que de la stabilité avérée (valeur seuil admise $=2,5$ )

\section{DISCUSSION}

I es deux vecteurs présents à Ambohimena étaient An. funestus et An. arabiensis.

1 Les densités agressives d'An. funestus observées semblent moins fortes que celles rapportées, une dizaine d'années plus tôt, par Ratefiarisoa et al. (1988), dans le bassin de Mahasolo. Le taux d'endophagie global n'était pas très élevé. Cette endophagie limitée a été signalée sur les Hautes-Terres par d'autres auteurs (Fontenille et al., 1989). Cette espèce est plutôt endoexophage. Une fraction non négligeable de femelles endophages a besoin de prendre un deuxième repas de sang pour achever son premier développement ovarien. En accord avec Gruchet (1962), nous pensons que la méthode des ampoules ovariennes de Polovodova laisse trop d'individus douteux dans la détermination de leur parité. Ainsi, nous ne sommes pas en mesure d'appliquer la formule de Coz et al. (1961) pour l'estimation des taux de survie des populations d'anophèles. Toutefois, nous pensons que le cycle trophogonique d'An. funestus est supérieur à deux jours dans cette région; que ce soit au cours de la saison chaude ou fraîche.

Les effectifs capturés dans les gîtes extérieurs proprement dits n'ont représenté qu'une faible proportion des captures. La fraction de la population fréquemment collectée au repos dans les dépendances extérieures pourrait être exophile au sens strict. An. funestus est une espèce endo-exophile (Grjebine, 1966). Elle manifeste une zoophilie et anthropophilie simultanée. Plus on s'éloigne de l'environnement humain et plus le taux d'anthropophilie baisse. Dans un contexte de lutte anti-vectorielle par pulvérisations intra-domiciliaires d'insecticide rémanent, dans le Moyen-Ouest malgache, il est particulièrement intéressant de noter la présence d'une fraction non négligeable d'An. funestus exophile et anthropophile en l'absence de pression insecticide. Les aspersions d'insecticides rémanents pourraient profitablement être étendus aux abris extérieurs. Du fait d'un contact plus élevé entre l'homme et le vecteur, mais aussi d'une meilleure longévité, nous avons observé que les populations d'An. funestus sont épidémiologiquement plus dangereuses à la fin du pre- mier tour (janvier) qu'à la fin du deuxième tour de riz (avril-mai). Là également, cette observation devrait être pris en compte dans l'établissement du calendrier des pulvérisations : pour une meilleure efficacité des traitements d'insecticides, la campagne de pulvérisation devrait commencer avant la période de transmission. L'infectivité d'An. funestus a été particulièrement faible. L'indice sporozoïtique n'est donc jamais élevé sur les Hautes-Terres, toujours inférieur à $1 \%$ (Blanchy et al., 1993). Sur les marges ouest des Hautes-Terres, cet indice ne semble pas plus élevé que dans les Hautes-Terres centrales. La transmission du paludisme par ce vecteur a été mise en évidence au cours de la saison chaude et humide et jusqu'au premier mois de la saison sèche. Dans le village étudié, An. funestus a été le vecteur principal, sinon le seul. La mise en évidence de différences comportementales (trophiques et/ou choix de lieu de repos) et d'infectivité pour $P$. falciparum laissent penser que plusieurs populations coexistent et pourraient présenter une structuration génétique. Cette question fera l'objet d'observations ultérieures.

Les densités agressives d'An. arabiensis pour l'homme restent faibles. Entre octobre et février, l'abondance maximale des adultes est liée à la productivité des gîtes larvaires. À cette période de l'année, les gîtes favorables sont représentés par les rizières labourées et/ou repiquées. Nos résultats sont en accord avec les enquêtes réalisées dans d'autres sites sur les Hautes-Terres malgaches. Comme Ratefiarisoa, en 1988, nous avons trouvé qu'An. arabiensis était délibérément exophage et présentait une forte tendance à l'exophilie (Fontenille et al., 1990; Laventure et al., 1996; Ratefiarisoa et al., 1988). Le taux d'anthropophilie est faible, de même que l'espérance de vie. Sur les Hautes-Terres, l'infectivité de cette espèce apparaît variable mais demeure faible de l'ordre de 0,06 \% (Ralisoa, 1996). Nous ne pouvons pas affirmer que l'indice sporozoïtique de cette espèce soit nul à Ambohimena, mais il est certainement très faible : probablement inférieur à $0,1 \%$. En résumé à Ambohimena, cette espèce présente une forte zoophilie et une forte exophagie. La longévité, le contact homme-vecteur et la densité sont restés faibles. La capacité vectorielle est donc particulièrement limitée. Le rôle d'An. arabiensis dans la transmission des plasmodies n'a pas été mis en évidence. Au total, on peut estimer que son rôle dans l'épidémiologie du paludisme est secondaire.

Dans le Moyen-Ouest et à 1000 mètres d'altitude, la transmission de $P$. falciparum est de type saisonnière longue et dure au moins cinq mois. Le taux d'inoculation annuel spécifique de $P$. falciparum présente une forte variation d'une année sur l'autre, mais la période où chaque vecteur pris isolément est épidémiologiquement le plus dangereux a été observée entre décembre et janvier : période de plus forte humidité. Rappelons que, pour des raisons d'abondance relative, 
cette période ne correspond pas au maximum de la transmission en mars-avril.

L'indice de stabilité est compris entre 0,5 et 2,5. La pénéplaine de Mandoto dans laquelle est situé le village étudié relève d'une zone de transition entre le paludisme stable et le paludisme instable. D'après Alles et al. (1998), l'impact des moyens de lutte antivectorielle n'a une incidence sur le nombre de cas cliniques qu'à partir du moment où le taux d'inoculation est inférieur à 10 piqûres infectantes/homme/an. Dans le Moyen-Ouest, nous avons toutes les raisons de croire que dans un contexte de stabilité non avérée et dans une région qui présente un taux d'inoculation annuel inférieur à 10 piqûres infectantes/homme/an, une intervention de lutte antivectorielle aurait un impact direct sur la morbidité palustre.

Nous proposons que les opérations de pulvérisations intra-domiciliaires soient étendues sur les marges occidentales des Hautes-Terres qui constituent actuellement un réservoir potentiel de vecteurs et de parasites. Dans cette perspective, la pulvérisation d'insecticide suffisamment rémanent devrait avoir lieu avant la période de transmission, soit début novembre, pour ces régions de la marge occidentale des Hautes-Terres qui pratiquent deux tours de riz.

\section{REMERCIEMENTS}

I es auteurs remercient Vincent Robert pour son aide lors de la rédaction du manuscrit. Les auteurs remercient également tous ceux qui ont contribué à la réalisation de cette recherche, en particulier G. Hébrard, J. Rakotoarisolo, E. Rakotondraibe, M. Rakotoniaina et D. Randrianambinina. Nous ne saurons oublier la parfaite et cordiale collaboration des habitants du village d'Ambohimena.

Cette étude a été réalisée dans le cadre du Programme RAMSE (Recherches Appliquées à Madagascar en Santé et Environnement) de l'Institut de Recherche pour le Développement (IRD) en collaboration avec la Direction de la Lutte contre les Maladies Transmissibles (DLMT) et l'Institut Pasteur de Madagascar (IPM). Elle a bénéficié d'un financement de l'OMS - projet IDA et de l'IRD.

\section{RÉFÉRENCES}

Alles H.K., MENDIS K.N. \& CARTER R. Malaria mortality rates in South Asia and in Africa: implications for malaria control. Parasitology Today, 1998, 14, 9.

Beier J.C., Wirtz R.A., Perkins P.V., Koros J., Diggs D., GaRgam T.P. \& KOECH D.R. Bloodmeals identification by direct enzyme-linked immunosorbent assay (ELISA), tested on Anopheles (Diptera: Culicidae) in Kenya. Journal of Medical Entomology, 1988, 25, 9-16.

Blanchy S., Rakotonjanabelo A., Ranaivoson G. \& RajaonaRIVELO E. Epidémiologie du paludisme sur les HautesTerres Malgaches depuis 1878. Cahiers Santé, 1993, 3, 155161.

Burkot T.R., Goodman W.G. \& Defoliart G.R. Identification of mosquito blood meals enzyme-linked immunosorbent assay. American Journal of Tropical Medicine and Hygiene, 1981, 30, 1336-1341.

Burkot T.R., Wiluiams J.L. \& Schneider I. Identification of Plasmodium falciparum-infected mosquitoes by a double antibody Enzyme-Linked Immunosorbent Assay. American Journal of Tropical Medicine and Hygiene, 1984, 33, 783788.

Coz J., Gruchet H., Chauvet G. \& Coz M. Estimation du taux de survie chez les anophèles. Bulletin de la Société de Pathologie Exotique, 1961, 54, 1353-1358.

Detinova T.S. Méthodes à appliquer pour classer par groupes d’âge les diptères présentant une importance médicale (monographie $n^{\circ}$ 47). Organisation Mondiale de la Santé, Genève, 1963, $220 \mathrm{p}$.

Donque D. Contribution géographique à l'étude du climat de Madagascar, Thèse d'État, Université d'Antananarivo, 1975, 478 p.

Fontenille D., Rakotoarivony I. \& Lepers J.P. Résultats entomologiques du programme paludisme de l'Institut Pasteur de Madagascar, sur les Hauts-Plateaux malgaches, en 1987-1988. Archives de l'Institut Pasteur de Madagascar, 1989, 56, 275-286.

Fontenille D., Lepers J.P., Campbell G.H., Coluzzi M., RakoTOARIVONY I. \& Collanges P. Malaria transmission and vector biology in Manarintsoa, High Plateaux of Madagascar. American Journal of Tropical Medicine and Hygiene, 1990, 43, 107-115.

Fontenille D. \& CampBell G.H. Is Anopheles mascarensis a new malaria vector in Madagascar? American Journal of Tropical Medicine and Hygiene, 1992, 46, 28-30.

GARETT-Jones C. The human blood index of malaria vector in relation to epidemiological assessment. Bulletin of the World Health Organisation, 1964, 30, 241-261.

Gillies M.T. \& Coetzee M. A supplement to the Anophelinae of Africa South of the Sahara (Afrotropical region), $n^{\circ} 55$. South African Institute for Medical Research, Johanesburg, 1987, $143 \mathrm{p}$.

GrJebine A. Insectes. Diptères Culicidae Anophelinae. Faune de Madagascar (fascicule $n^{\circ} 22$ ), Muséum National d'Histoire Naturelle, Paris, 1966, 487 p.

Gruchet H. Étude de l'âge physiologique des femelles d'Anopheles funestus Giles dans la région de Miandrivazo, Madagascar. Bulletin de la Société de Pathologie Exotique, 1962, 55, 165-174.

Laventure S., Mouchet J., Blanchy S., Marrama L., Rabarison P., Andrianaivolambo L., Rajaonarivelo E., Rakotoarivony I. \& Roux J. Le riz, source de vie et de mort sur les plateaux de Madagascar. Cahiers Santé, 1996, 6, 79-86.

MacDonald G. The epidemiology and control of malaria. Oxford University, London, 1957, 201 p. 
Marrama L., Laventure S., Rabarison P. \& Roux J. Anopheles mascarensis (De Meillon, 1947) : vecteur principal du paludisme dans la région de Fort-Dauphin (Sud-est de Madagascar). Bulletin de la Société de Pathologie Exotique, 1999, 92, 136-138.

Mouchet J. \& Blanchy S. Diversité et stratification du paludisme à Madagascar. Cahiers Santé 1995, 5, 386-388.

Mutrhead-Thomson R.C. A pit shelter for sampling outdoor mosquito populations. Bulletin of the World Health Organisation, 1958, 19, 241-261.

Ralisoa O. Répartition des vecteurs du paludisme dans les différentes strates écologiques. In : Effets sanitaires de l'irrigation et des aménagements hydro-agricoles : le cas de Madagascar. Research Centre for International Agrarian and Economic Development, Heidelberg, 1996, 51-61.

Ratefiarisoa R.F., Randrianasolo R.O. \& Rajaonarivelo E. Sur la faune anophélienne et les vecteurs principaux du paludisme de la région de Mahasolo (Moyen-Ouest de Madagascar). Recherches pour le Développement, Série Sciences biologiques, 1988, 6, 79-93.

Scott J.A., Brogton W.G. \& Collins F.H. Identification of single specimen of Anopheles gambiae complex by the polymerase chain reaction. American Journal of Tropical Medicine and Hygiene, 1993, 49, 520-529.

Wirtz R.A., Zavala F., Charoenvit Y., Campbell G.H., Burkot T.R., Schneider I., Esser K.M., Beaudouin R.L. \& ANdré R.G. Comparative testing of monoclonal antibodies against Plasmodium falciparum sporozoites for ELISA development, Bulletin of the World Health Organisation, 1987, 65, $39-45$.

Reçu le 12 mai 2003

Accepté le 27 octobre 2003 